\title{
Surgical Strategy in Bouveret's Syndrome. A Case Report
}

\author{
Dénes Márton István, Török Árpád, Éltes Etele, Kucserik Levente, András Zsolt, Borz Bogdan, Borz \\ Cristian*
}

University of Medicine and Pharmacy Targu Mures, Romania

Bouveret's syndrome is a high mechanical obstruction due to impaction of a gallstone into the duodenum, through a cholecystoduodenal fistula. It belongs to a larger group of gallstone ileus, a disease which occurs after developing a fistula between the gallbladder and the gastrointestinal tract. This is a rare complication of gallstones but because it appears in elderly people, it has a high morbidity and mortality. Patients have various symptoms and the treatment is individualized. We present here a case of a 67 years old patient, admitted in emergency, presenting symptoms of high bowel obstruction, with onset 5 days before admission. Abdominal ultrasound reveals a $5 \mathrm{~cm}$ stone that seems to be in the gallbladder, gastric stasis and at gastroscopy appears a foreign body impacted in the duodenum. After a short preparation the patient underwent surgery. We found a dilated stomach and a large cholecystoduodenal fistula with an impacted gallstone in the duodenum. We performed one stage surgery: cholecystectomy, extraction of the stone and suturing of the fistula. We reestablished the continuity of the intestinal tract. We performed also an ileostomy for feeding the patient and protecting the anastomoses. The postoperative evolution was favorable. We consider that one stage surgical treatment in gallstone ileus is an option, if the patient is in good condition and we have an adequate postoperative intensive care management.

Keywords: bilio-enteric fistula, gallstone ileus, gastric outlet obstruction

Received: 14 October 2015 / Accepted: 20 March 2016

\section{Introduction}

Gallstone ileus is a rare complication of gallstones. It is an ileus of biliary etiology produced by the impaction of a gallstone in the gastrointestinal tract. It is a rather rare form of bowel obstruction, representing 1-3\% of all cases [1]. Bouveret's syndrome, a rare condition, appears when the site of the gallstone obstruction is high in the duodenum and causes gastric outlet obstruction with high morbidity and mortality [2]. This condition is possible because of the creation of a fistula between the inflamed gallbladder and the adjacent part of the intestine. The acute inflammation produces adhesions between the gallbladder and the gastrointestinal tract. The pressure made by the stone on the tissue ends with necrosis of the wall of the two adjacent structures, permitting the stone to penetrate the intestine. The most common site of impaction is in the terminal ileum (60-70\%). In general, bigger stones cause proximal obstruction. The prevalence of this disease decreased dramatically the last 25 years thanks to early diagnosis of gallstones by ultrasound and laparoscopic cholecystectomy [3]. In Bouveret's syndrome, because unspecific symptoms, usually the diagnosis is delayed. Also the treatment of this condition remains controversial. In this paper we present the surgical treatment of a patient with Bouveret's syndrome admitted in our department and a short review of the literature. The treatment has to be customized, adapted to the condition of the patient and the intraoperative findings.

\section{Case report.}

A 67 year old patient was admitted in emergency conditions in our department, presenting abdominal cramps, nausea and vomiting foods eaten 2-3 days ago. The onset of the symptoms was brutal, 5 days earlier accompanied by debility and weight loss. In her past history the patient had repeatedly cramps in her right flank, nausea and vomiting. An ultrasound examination revealed gallstone, 3 years ago. The woman was programed for elective cholecystectomy but failed to have the surgery done. In the last year her general condition deteriorates constantly, losing weight $(20 \mathrm{~kg})$, and experiencing loss of appetite. She had diabetes mellitus type 2. Local examination revealed an upper right quadrant pain. The blood pressure was $100 / 60 \mathrm{mmHg}$ and a heart rate of $110 / \mathrm{min}$. Laboratory tests were modified: urea $160 \mathrm{mg} \%$, creatinine $1.47 \mathrm{~g} \%$, erythrocyte sedimentation rate $150 / 115$, INR 1.34 .

The X-ray examination did not show signs of bowel obstruction. The abdominal ultrasound showed a $5 \mathrm{~cm}$ gallstone and air in the biliary tract. Through the nasogastric tube $1500 \mathrm{ml}$ of gastric fluid were removed. At gastroscopy the stomach appears enlarged and a foreign body impacted in the duodenum is visualized. After a short intensive care management, the patient underwent surgery. Through a midline laparotomy we discovered adhesions between the gallbladder and duodenum and a large cholecystoduodenal fistula (fig. 1,2) with a $5 \mathrm{~cm}$ gallstone impacted in the duodenum (fig. 3). The stomach was dilated with thick walls. We broke down the fistula and extracted with difficulty the impacted stone. Due to adhesions and inflamed tissue around the common bile duct, the cholecystectomy was 


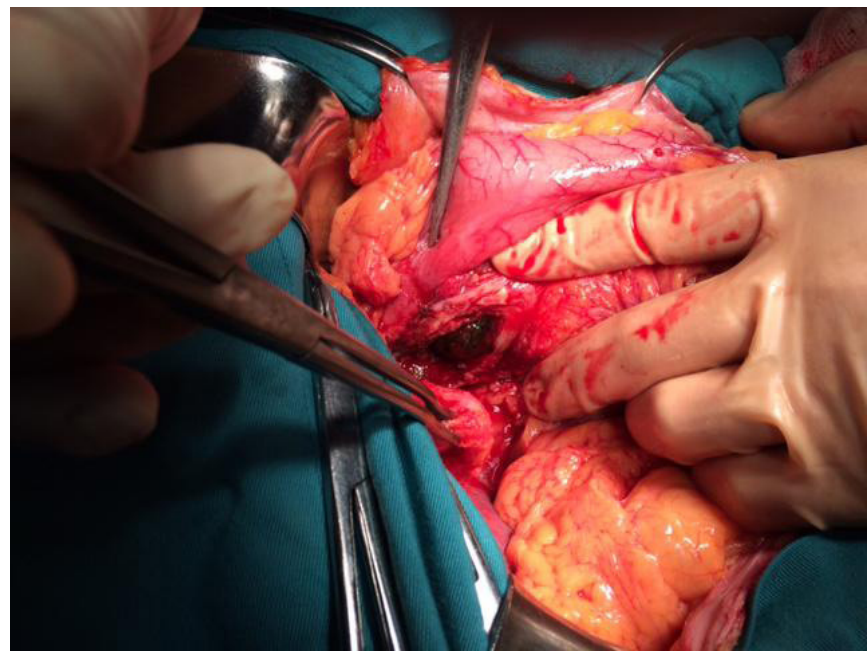

Fig.1. The gallstone impacted in the duodenum

time consuming. The large defect in the duodenal wall, in close proximity of the common bile duct didn't permit us a safely suture of the duodenum. In order to avoid lesions of the common bile duct and to prevent a duodenal fistula after sewing its wall, we decided to divide, to sever the bile duct and close the distal stump. We repaired the duodenal defect by suturing it to a Roux en Y jejunal limb (latero-lateral duodeno-jejunostomy with 3.0 monofilament continuous suture) (fig. 4). Further, on the same jejunal segment, we restored the biliary system continuity by termino-lateral hepatico-jejunostomy (with 4.0 monofilament continuous suture), protected with a transanastomotic tube in Volker manner (fig. 4). To put the anastomoses on rest and for feeding the patient, we performed a Stamm jejunostomy (fig. 5). The patient received also intensive care management. The postoperative course was uneventful until the $6^{\text {th }}$ day when suddenly through the contact drain flowed approximately $700 \mathrm{ml}$ of gastric liquid. At reoperation we found a small fistula at the hepatico-jejunal anastomosis without peritonitis. We decided to suture the defect and to place another contact drain. This time, the postoperative course was favorable, the patient being fed through

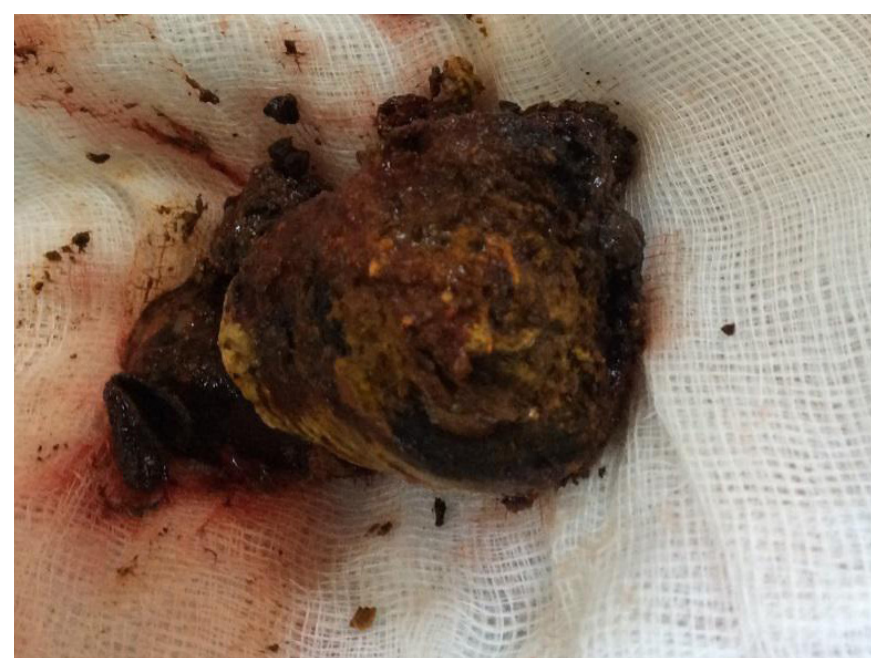

Fig. 3. The removed gallstone

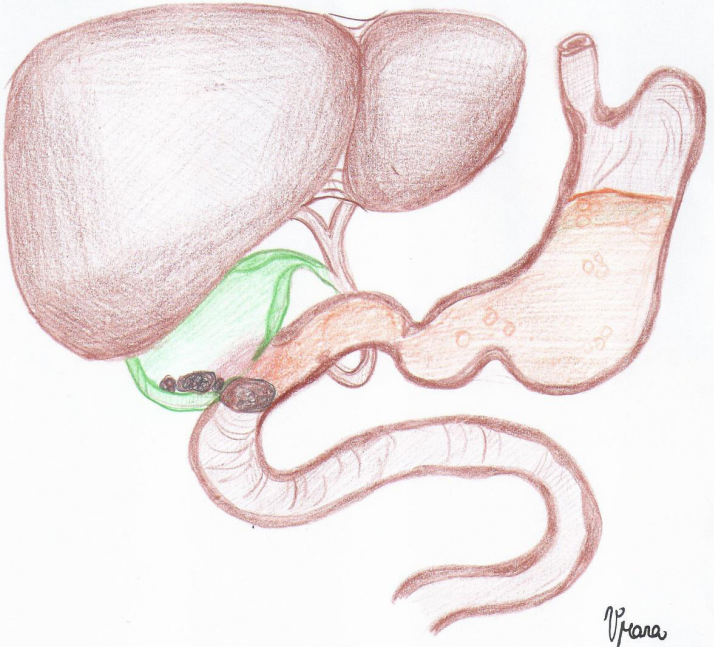

Fig. 2. Illustration of the cholecystoduodenal fistula

the jejunostomy for 7 days. Through the transanastomotic tube the daily output was $200 \mathrm{ml}$ of bile. The patient was discharged after 14 days in good condition. The transanastomotic tube was removed after 4 weeks.

\section{Discussion}

Biliary ileus is a rare complication of gallstone disease (cholelithiasis). The stone enters the intestinal tract through a cholecystodigestive fistula and occludes the lumen of the small bowel, developing an intestinal obstruction. The first case of biliary ileus was described by Bartolini in 1654 but the complete depiction of the disease was made by Coirvoiser in 1980, reporting 131 operated cases with a $44 \%$ mortality. The fistula accounts for $0.3-5 \%$ of cases with cholelithiasis $[1,2]$, the most frequent being the cholecystoduodenal fistula (70\%), followed by the cholecystocolic fistula (10-20\%). The less frequent is the cholecystogastric fistula (4\%).

This condition occurs especially in elderly women, between 65-75 years. During an acute cholecystitis the inflammation is followed by adhesions between the gallbladder and the small bowel. The high pressure within the

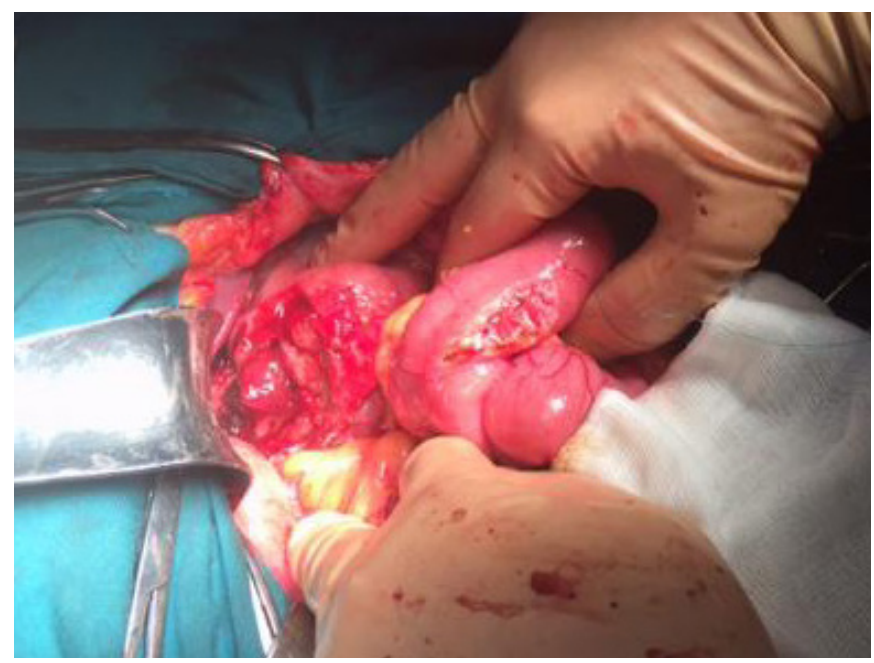

Fig. 4. Preparing the L-L duodenojejunostomy 


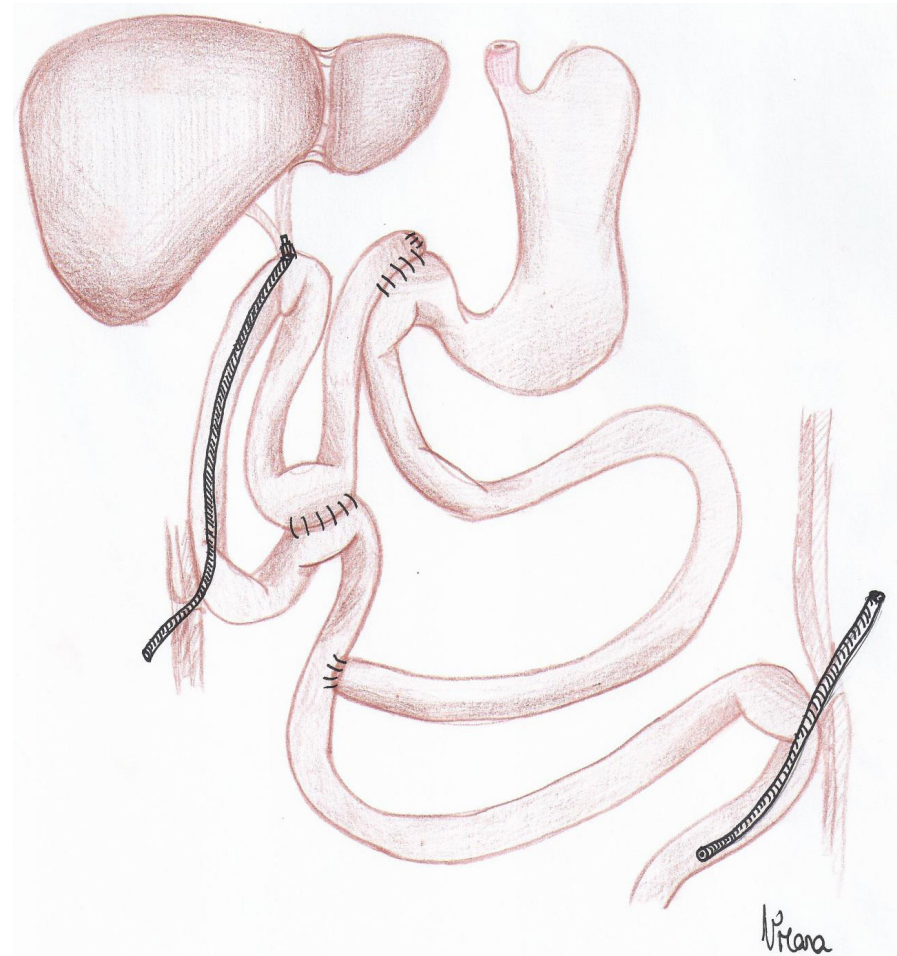

Fig. 5. Illustration of the surgical procedure

bladder, due to obstruction, leads to ischemia and necrosis, initiating the fistula. Through the fistula, the gallstone (usually a large one) passes into the intestine and becomes impacted in a narrow segment of the small bowel. In 50$75 \%$ of cases this place is represented by the terminal ileum, in $20-40 \%$ of cases the proximal ileum and jejunum, in less than $10 \%$ of cases the duodenum and seldom the colon [4]. The size of the gallstones varies between 2 and $5 \mathrm{~cm}[5]$.

Bouveret's syndrome described by the author in 1896, is related to impaction of the stone in the stomach or duodenum, leading to a gastric outlet obstruction [6]. Women are affected more often, especially elderly patients (mean age is 74 years) [2].

Preoperative diagnosis is difficult, in half of the cases the condition being discovered during surgery. Most of the affected patients have important comorbidities so the mortality is high. The classic triad of Rigler [6]: mechanical bowel obstruction, air in the biliary tract and the presence of the stone in the small bowel is not always encountered. Most common symptoms are: epigastric pain, nausea and vomiting [7]. Patients also report bloating, dehydration, upper digestive bleeding and heartburn [8]. In 15\% of cases the stones can be seen on X ray or CT scan [1]. Differential diagnosis is made with perforated ulcer, pancreatitis, gastric volvulus, malignant fistula and foreign bodies [9]. It is recommended to begin the imaging tests with an abdominal ultrasound that can reveal the cholecystitis and the dilated stomach. The CT scan shows the inflammation of the tissues, the fistula and the stones. In 69\% of cases at duodenoscopy we can see undigested food in the dilated stomach, duodenal ulcer with inflammation and edema or even the fistula.

The treatment for biliary ileus remains controversial. Many authors recommend the endoscopic or percutaneous method before the surgical intervention $[2,10,11]$, because we are dealing with elderly patients, with multiple comorbidities, which can lead to intra- and postoperative complications. Endoscopic or percutaneous treatment methods are: mechanical lithotripsy, laser lithotripsy, extracorporeal shock wave lithotripsy and intracorporeal electrohydraulic lithotripsy. However in $91 \%$ of the cases, the surgical treatment remains the treatment of choice. The size of the stone has an important role because stones that measure more than $2.5 \mathrm{~cm}$ are very difficult to remove by the endoscope. The preoperative management of the patient is essential, with special attention towards the hydroelectrolytic balance and the treatment of associated diseases [5]. There is not a consensus regarding the surgical procedure. The options are: 1 . enterolitotomy; 2 . enterolitotomy with cholecystectomy afterwards (two-stage surgery); 3. enterolitotomy with cholecystectomy and closing of the fistula (one-stage surgery). The clear advantage of the one-stage surgery is the definitive character of the treatment, even though a notable morbidity and mortality was demonstrated (7-25\%) [3]. On the contrary, the advantage of the two-stage surgery is that it initially solves the intestinal obstruction emergency and then in the second stage, the cholecystoduodenal fistula, this way, lowering the mortality rate. The stone extraction as the first step of the two stage surgery has a $11.7 \%$ mortality rate, compared to the $16.9 \%$ mortality rate of the one-stage surgery. Cases with spontaneous closure of the fistula after the stone removal have been reported [5]. In the literature there are described 3 reasons to treat the cholecystoenteric fistula: the relapse of the obstruction, recurrent biliary infections and the risk to develop a cholangicarcinoma. This risk is 15 folds higher in patients with fistula by comparison with the rest of the population [3]. This are the reasons why the management of Bouveret's syndrome remains controversial and must be individualized according to the age and general state of the patient but also depending on the experience of the surgical school. The biliary ileus has a poor prognosis, the mortality rate is around $20 \%$ [5].

\section{Conclusions}

Bouveret's syndrome is an extremely rare complication of the gallstone disease, with a high mortality rate. The preoperative diagnosis requires a high level of presumption and a good clinical vision. Duodenoscopy helps early diagnosis, enabling a fast surgical intervention. The surgical treatment must be individualized. The choice of surgical procedure requires a holistic vision of the patient's problems, a comparison of the cost-benefit ratio for one-stage surgical intervention that definitively solves the illness, or to adopt a two-stage surgery. In the literature there is an emphasis towards the one-stage surgery especially in cases of patients 
that can be properly stabilized in the preoperative period, when local and general conditions enable a prolonged surgical procedure.

\section{Conflict of interest}

None to declare.

\section{References}

1. Pezzoli A, Maimone A, Fusetti N, Pizzo E. Gallstone ileus treated with non-surgical conservative methods: a case report. Journal of Medical Case Reports 2015;9:15.

2. Nickel F, Müller-Eschner MM, Chu J, von Tengg-Kobligk H, Müller-Stich BP. Bouveret's syndrome: presentation of two cases with review of the literature and development of a surgical treatment strategy. BMC Surgery 2011;13:33.

3. Donati M, Cardi F, Brancato G, Calo P, Donati A. The surgical treatment of a rare complication: gallstone ileus. Ann. Ital. Chir. 2010;81:57-62

4. M.R. Attri, Shahnawaz Ahangar, Rajni Bhardwaj, Cholecystoduodenal
Fistula: An Intraoperative Diagnosis. Journal of Medical Education@ Research. 2010;12(1)

5. Nuño-Guzmán CM, Arróniz-Jáuregui J, Moreno-Pérez PA, et al. Gallstone ileus: One-stage surgery in a patient with intermittent obstruction. World J Gastrointest Surg 2010;2(5): 172-176

6. Sağlam F, Sivrikoz E, Alemda A, Kamalı S, Arslan U, Güven H. Bouveret syndrome: A fatal diagnostic dilemma of gastric outlet obstruction. Ulus Travma Acil Cerrahi Derg 2015;21(2):157-9.

7. Brennan GB, Rosenberg RD, Arora S. Bouveret syndrome. Radiographics 2004:24:1171-1175

8. Koulaouzidis A, Moschos J. Bouveret"s syndrome. Narrative review. Ann Hepatol 2007:6(2):89-91

9. Dan D, Collure DW, Hoover EL. Bouveret"s sundrome: revisiting gallstone obstraction of the duodenum. J Natl Med Assoc 2003;95(10):969-973

10. Doyceva I, Limaye A, Suman A, Forsmark CE, Sultan S. Bouveret"s syndrome: case report and review of the literature. Gastroenterol Res Pract 2009;2009:914951

11. Huebner ES, DuBois S, Lee SD, Saunders MD. Successful endoscopic treatment of Bouveret!s syndrome with intracorporeal electrohydraulic lithotripsy. Gasrointest Endosc 2007;66(1):183-184 